\title{
Linseed hydrogel-mediated green synthesis of silver nanoparticles for antimicrobial and wound-dressing applications
}

This article was published in the following Dove Press journal:

International Journal of Nanomedicine

6 April 2017

Number of times this article has been viewed

\author{
Muhammad Tahir Haseeb ${ }^{1,2}$ \\ Muhammad Ajaz Hussain ${ }^{3}$ \\ Khawar Abbas ${ }^{3}$ \\ Bahaa GM Youssif ${ }^{4,5}$ \\ Sajid Bashir \\ Soon Hong Yuk ${ }^{2}$ \\ Syed Nasir Abbas Bukhari ${ }^{5}$ \\ 'Faculty of Pharmacy, University of \\ Sargodha, Sargodha, Pakistan; ${ }^{2}$ College \\ of Pharmacy, Korea University, Sejong, \\ Republic of Korea; ${ }^{3}$ Department of \\ Chemistry, University of Sargodha, \\ Sargodha, Pakistan; ${ }^{4}$ Department of \\ Pharmaceutical Organic Chemistry, \\ Faculty of Pharmacy, Assiut University, \\ Assiut, Egypt; ${ }^{5}$ Department of \\ Pharmaceutical Chemistry, College \\ of Pharmacy, Aljouf University, Aljouf, \\ Sakaka, Saudi Arabia
}

Correspondence: Muhammad

Ajaz Hussain

Department of Chemistry, University of Sargodha, Sargodha 40I00, Pakistan

Tel +9234686I 4959

Fax +92 483222121

EmailmajazI72@yahoo.com

Bahaa GM Youssif

Department of Pharmaceutical Organic Chemistry, Faculty of Pharmacy, Assiut

University, Assiut 71526, Egypt

Tel +20 I00 4237882

Email bgyoussif@ju.edu.sa
Abstract: Polysaccharides are being extensively employed for the synthesis of silver nanoparticles (Ag NPs) having diverse morphology and applications. Herein, we present a novel and green synthesis of Ag NPs without using any physical reaction conditions. Linseed hydrogel (LSH) was used as a template to reduce $\mathrm{Ag}^{+}$to $\mathrm{Ag}^{0}$. $\mathrm{AgNO}_{3}(10,20$, and $30 \mathrm{mmol}$ ) solutions were mixed with LSH suspension in deionized water and exposed to diffused sunlight. Reaction was monitored by noting the change in the color of reaction mixture up to $10 \mathrm{~h}$. Ag NPs showed characteristic ultraviolet-visible (UV/Vis) absorptions from 410 to $437 \mathrm{~nm}$ in the case of sunlight and $397-410 \mathrm{~nm}$ in the case of temperature study. Transmission electron microscopy images revealed the formation of spherical Ag NPs in the range of 10-35 nm. Face-centered cubic array of Ag NPs was confirmed by characteristic diffraction peaks in powder X-ray diffraction spectrum. Ag NPs were stored in LSH thin films, and UV/Vis spectra recorded after 6 months indicated that Ag NPs retained their texture over the storage period. Significant antimicrobial activity was observed when microbial cultures (bacteria and fungi) were exposed to the synthesized Ag NPs. Wound-healing studies revealed that Ag NP-impregnated LSH thin films could have potential applications as an antimicrobial dressing in wound management procedures.

Keywords: silver nanoparticles, green synthesis, antimicrobial studies, wound dressing, storage and stability

\section{Introduction}

Metal-based nanoparticles (NPs) have intrigued material scientists in the fields of applied and fundamental research for the last two decades. ${ }^{1}$ Unique electronic, ${ }^{2}$ catalytic, ${ }^{3}$ and optical ${ }^{4}$ properties of such metal NPs have led to their potential applications in bioconjugation, biosensing, ${ }^{5,6}$ and surface-enhanced Raman scattering. ${ }^{7}$ Although NPs have been synthesized from a large variety of metals, silver nanoparticles (Ag NPs) have gained special importance over the last few years. Ag NPs have a wide range of potential applications in lithography, ${ }^{8}$ microelectronics,${ }^{9,10}$ supermagnets, ${ }^{11}$ and semiconductors. ${ }^{12}$ Silver is also known to show antimicrobial properties, and several silver salts and their derivatives have been used as commercial antimicrobial agents. ${ }^{13,14}$ Recently, Ag NPs have been employed as antimicrobial agents in water filters ${ }^{15}$ and cotton fabrics. ${ }^{16}$

Owing to a large plethora of applications of Ag NPs, a number of methods have been devised for their synthesis. The more commonly used methods include chemical and photochemical reduction, ${ }^{17,18}$ electrochemical method, ${ }^{19}$ and laser ablation. ${ }^{20,21}$

Use of chemical reduction method for synthesis of Ag NPs involves environmentally hostile reducing agents, ${ }^{22,23}$ toxic solvents, ${ }^{24}$ and nonbiodegradable/synthetic 
stabilizing agents. ${ }^{25,26}$ Such synthesis protocols can pose a serious threat to environment and also cause biological hazards. Further, high surface area-to-volume ratio of Ag NPs causes them to cling together with passage of time, thereby limiting their potential applications. Therefore, there has been keen interest to explore low-cost, biodegradable, nontoxic, and green agents ${ }^{27,28}$ for synthesis of Ag NPs. ${ }^{29,30}$

Recently, polysaccharides are gaining importance as green reducing and self-capping agents for synthesis of $\mathrm{Ag}$ NPs. ${ }^{31,32}$ The film-forming properties of polysaccharides can be additionally used for preventing the coagulation of $\mathrm{Ag}$ NPs over long-term storage. ${ }^{33}$ Starch- and chitosan-based Ag $\mathrm{NPs}^{34,35}$ have been synthesized but it was difficult to produce highly concentrated Ag NPs and store them for a longer period without getting agglomerated. Therefore, a need was felt to look for polysaccharides capable of producing highly concentrated and stable Ag NPs. In such an attempt, we have utilized linseed hydrogel (LSH) as a green reducing and selfcapping agent for the formation of Ag NPs. LSH is composed of two major polysaccharidal fractions: rhamnogalacturonan and arabinoxylan. ${ }^{36,37}$ The effect of $\mathrm{AgNO}_{3}$ concentration, reaction time, and reaction temperature on the formation of Ag NPs is also studied. We are further focused to study antimicrobial properties of the pristine Ag NPs. Wound-healing capability of the synthesized Ag NPs will also be evaluated. The potential of LSH thin films as a storage medium for the Ag NPs is being reported for the first time.

\section{Experimental Materials}

Linseed used for hydrogel extraction was purchased from local market. $\mathrm{AgNO}_{3}$ was obtained from Sigma-Aldrich, St Louis, MO, USA. Analytical grade solvents and other reagents were procured from Fluka (Sigma-Aldrich) and used as received. Deionized water was used for preparation of all solutions and isolation of LSH.

\section{Isolation of LSH}

LSH was conveniently isolated using hot water extraction. ${ }^{38}$ Linseed (100 g) was thoroughly washed with deionized water to clean it from dust particles and soaked overnight in deionized water $(1,000 \mathrm{~mL})$. The swollen seeds were heated at $80^{\circ} \mathrm{C}$ for $30 \mathrm{~min}$ under stirring. Hydrogel extruded from these seeds was separated with the help of nylon gauze. LSH was washed with $n$-hexane to clean it from waxy or fatty material. LSH was further washed with deionized water and separated after centrifugation. Afterwards, it was dried under air for $24 \mathrm{~h}$ and then placed in vacuum oven at $60^{\circ} \mathrm{C}$ for the next $24 \mathrm{~h}$. Fine powder of LSH was collected after passing through stainless-steel mesh no 60 and stored in an air-tight container in a desiccator.

\section{Preparation of $\mathrm{AgNO}_{3}$ solution}

To prepare $\mathrm{AgNO}_{3}$ solution (10 mmol), $\mathrm{AgNO}_{3}(169.87 \mathrm{mg}$ ) was dissolved in deionized water (100 mL). Similarly, 20 and $30 \mathrm{mmol}$ solutions of $\mathrm{AgNO}_{3}$ were prepared by dissolving 339.74 and $509.61 \mathrm{mg}$ of $\mathrm{AgNO}_{3}$ in deionized water, respectively.

\section{Preparation of LSH suspension}

LSH powder $(0.2 \mathrm{~g})$ was suspended in deionized water $(10 \mathrm{~mL})$ to prepare $2 \%(\mathrm{w} / \mathrm{v})$ solution.

\section{$\mathrm{LSH}$-mediated green synthesis of Ag NPs}

To synthesize Ag NPs, freshly prepared solution of $\mathrm{AgNO}_{3}$ (10 mmol, $10 \mathrm{~mL}$ ) was added to the suspension of LSH $(10 \mathrm{~mL})$. The mixture was stirred in a dark area for $5 \mathrm{~min}$ at room temperature to ensure homogeneous mixing of $\mathrm{AgNO}_{3}$ with LSH suspension. This mixture was then placed in sunlight for $10 \mathrm{~h}$, and progress of reaction was monitored periodically by observing the change of color. Sample $(1 \mathrm{~mL})$ of this reaction mixture was also analyzed with the help of ultraviolet-visible (UV/Vis) spectrophotometry. Same protocol was adopted for the other $\mathrm{AgNO}_{3}(20$ and $30 \mathrm{mmol}$ ) solutions.

\section{Film formation of LSH containing embedded Ag NPs}

A mixture of $\mathrm{AgNO}_{3}(10,20$, and $30 \mathrm{mmol})$ and LSH was exposed to diffused sunlight for $10 \mathrm{~h}$. Mixture was air dried in dark in a petri dish to produce LSH thin films containing the embedded Ag NPs and stored in an air-tight container. After 6 months, dry film was dissolved in deionized water, and the resultant suspension was characterized through UV/Vis spectrophotometry. Furthermore, Ag NPs were isolated from this suspension after centrifugation and then characterized by powder X-ray diffraction (PXRD) and transmission electron microscopy (TEM).

\section{UV/Vis spectrophotometry}

Formation of Ag NPs with the passage of time was monitored by UV/Vis spectrophotometry. Sample $(1.0 \mathrm{~mL})$ of the reaction mixture was taken at selected time intervals $(0.25$, $0.5,0.75,1,2,4,6,8$, and $10 \mathrm{~h}$ ) and analyzed in a range 200-800 nm on UV/Vis spectrophotometer (Optizen POP, Mecasays, Korea). The effect of various reaction conditions 
(concentration of $\mathrm{AgNO}_{3}$ solution and reaction time) on the progress of reaction was also studied. Moreover, effect of temperatures $\left(50,75\right.$, and $\left.100^{\circ} \mathrm{C}\right)$ on the synthesis of $\mathrm{Ag}$ NPs was also observed over a time period of $8 \mathrm{~h}$. In a temperaturedependent study, the mixture of $\mathrm{AgNO}_{3}$ solution and $\mathrm{LSH}$ suspension was placed at different temperatures in a dark area to prevent any photochemical reaction.

\section{Powder X-ray diffraction}

LSH suspension containing Ag NPs was freeze dried and characterized by PXRD. The PXRD spectra were recorded on a Bruker D8 Advance diffractometer (Bruker, Karlsruhe, Germany) (over a range of $10^{\circ} \mathrm{C}-80^{\circ} \mathrm{C}, 2 \theta$ ), operated at $40 \mathrm{~mA}$ and $40 \mathrm{kV}$.

\section{Transmission electron microscopy}

Morphology of Ag NPs was assessed by TEM. For this purpose, Ag NPs were isolated from solution by centrifugation and analyzed on a Philips 420 instrument. For recording TEM images, samples were drop casted on carbon-coated copper-wired TEM grid, and the instrument was operated at $120 \mathrm{kV}$. The LSH thin films were air dried in dark and stored for 6 months. After the specified storage period, Ag NPs were again analyzed by TEM. In a typical experiment, the stored LSH thin film impregnated with Ag NPs was dissolved in deionized water, and Ag NPs were isolated by centrifugation and viewed by TEM.

\section{Antimicrobial activity of Ag NPs}

Aqueous solution of the synthesized Ag NPs $(10 \mathrm{mg} / \mathrm{mL})$ was used to study the antimicrobial activity against different bacterial (Gram positive and Gram negative) and fungal strains. The tested bacterial strains include Streptococcus mutans American-type culture collection (ATCC) 25175, Staphylococcus epidermidis ATCC 12228, Pseudomonas aeruginosa ATCC 27853, Escherichia coli ATCC 25922, Staphylococcus aureus ATCC 25923, Bacillus subtilis ATCC 6633, and Actinomyces odontolyticus ATCC 17929. The fungal strain used in the antimicrobial assay was Aspergillus niger. Mueller Hinton Agar Media (Thermo Fisher Scientific, Waltham, MA, USA) was used as a growth medium for bacterial species, while fungi were grown on Sabouraud Dextrose Agar (Hardy Diagnostics, Santa Maria, CA, USA). Inoculums (fungal and bacterial culture in respective media, $10 \mathrm{~mL}$ ) were incubated at $27^{\circ} \mathrm{C}-30^{\circ} \mathrm{C}$ for $30-37 \mathrm{~h}$ for fungal strains and at $37^{\circ} \mathrm{C}$ for $24 \mathrm{~h}$ for bacteria. Fungal culture (7 days old) was washed, suspended in normal saline solution, and incubated at $28^{\circ} \mathrm{C}$ after being filtered through aseptic glass wool. The turbidity of inoculums was adjusted by $0.5 \mathrm{Mc}$ Farland Standard.
Disk diffusion method ${ }^{39}$ was used to determine the antimicrobial activity of Ag NPs. In a typical experiment, aqueous solution of the Ag NPs $(20 \mu \mathrm{L})$ was loaded/impregnated onto filter paper discs (Whatman filter paper no 1, $6 \mathrm{~mm}$ in diameter) and implanted on the surface of the microbial culture plates. These plates were then incubated at $37^{\circ} \mathrm{C}$ for $24 \mathrm{~h}$ for bacterial strains and at $30^{\circ} \mathrm{C}$ for $36 \mathrm{~h}$ for fungal strains. At the end of incubation period, zone of inhibition was measured in millimeter. Discs loaded with pure dimethyl sulfoxide and distilled water were used as negative control while $\mathrm{AgNO}_{3}$ solution $(20 \mathrm{mmol})$ was used as positive control. All experiments were performed in triplicate and mean values are reported.

\section{Designing of a wound-dressing patch}

LSH thin layer containing the embedded Ag NPs was evaluated as a wound-dressing material. Dressing patch consists of two layers. One layer is a cubic porous adhesive (United States Pharmacopeia, Rockville, MD, USA) backing membrane $(4 \times 4 \mathrm{~cm})$ and the second layer is cubic sterile cotton patch $(2 \times 2 \mathrm{~cm})$ immersed in LSH impregnated with Ag NPs (20 mmol, $0.5 \mathrm{~mL}$ ). This moist antiseptic patch was implanted at the center of adhesive membrane as shown in Figure 1B. This adhesive membrane will help the dressing patch to stick to the body. Freshly prepared patch was used during wound-healing studies.

\section{Wound-healing studies}

Nine healthy male rabbits $(2.5 \pm 0.2 \mathrm{~kg}, 4$ months old $)$ were selected and divided into three equal groups: control group, standard group, and test group. All rabbits were caged under standard laboratory conditions for $48 \mathrm{~h}$ before experiment and provided standard food and water ad libitum throughout the study. Rabbits were given anesthesia and placed on surgical table in natural position. Hair from the rear leg of the rabbit was shaved and excised a circular wound of $1 \mathrm{~cm}$ in diameter using a biopsy forceps. Wound of control group rabbits was left open and untreated. A Band-aid ${ }^{\circledR}$ dressing was applied to the wound of standard group while the test group was applied with dressing patch made as described above. The healing attributes were studied by tracing the raw wound area on tracing paper till the wound was completely epithelialized. ${ }^{40}$ An illustration of wound-healing procedure is shown in Figure 1. The study protocol was approved by Pharmacy Research Ethics Committee of University of Sargodha, Sargodha, Pakistan (Ref no 75-2014/PREC) and in accordance with the National Institute of Health's Guidelines for the Care and Use of Laboratory Animals. 

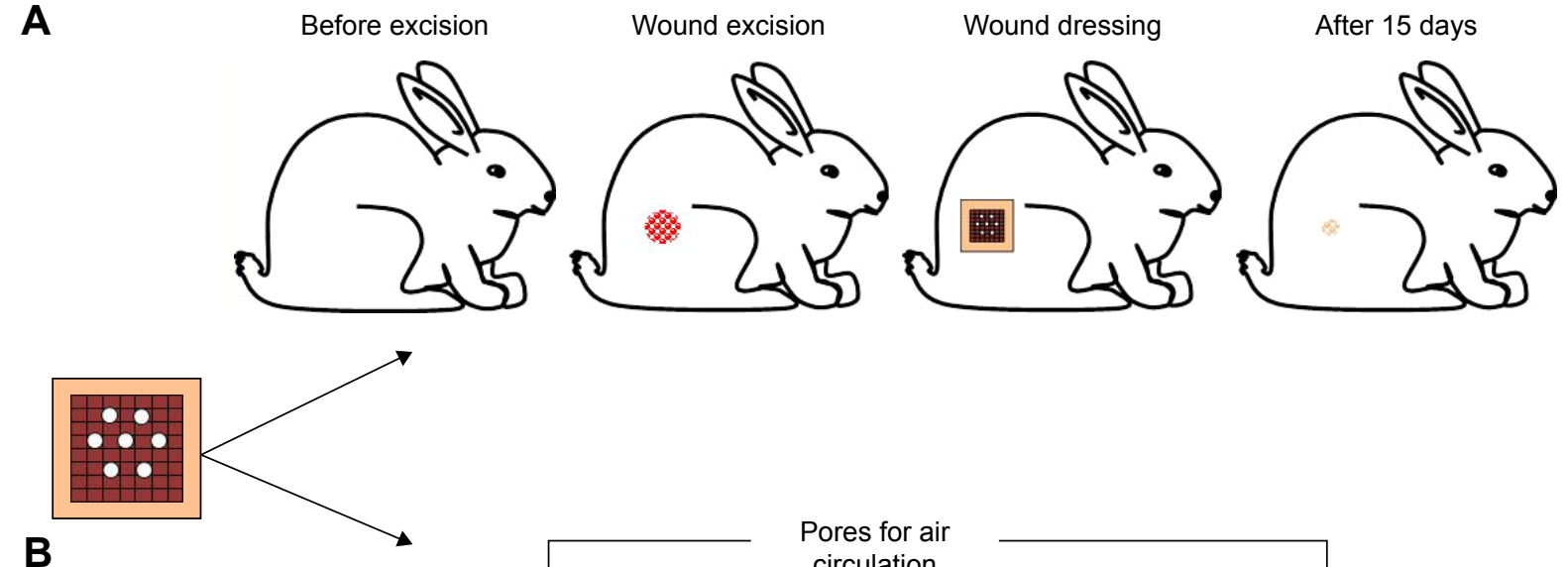

B

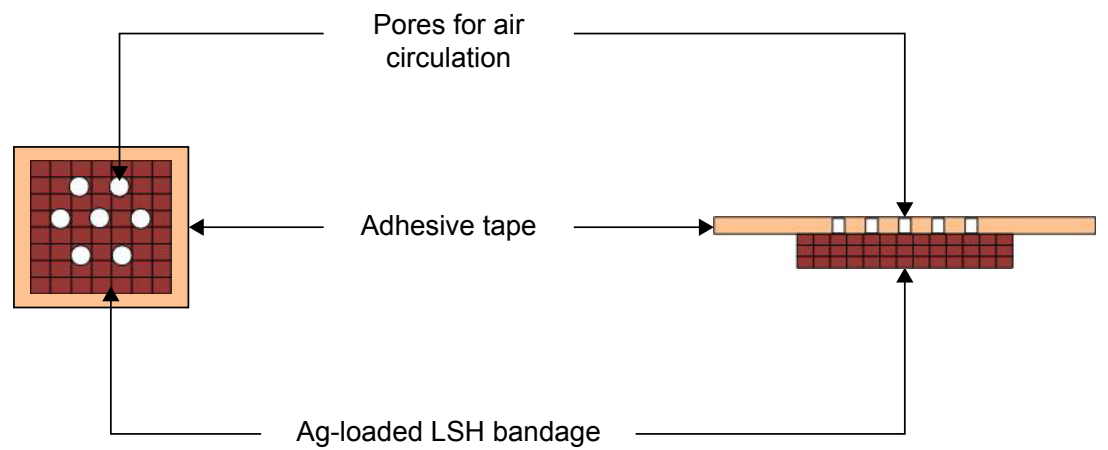

Figure I Schematic illustrations of wound treatment (A) and LSH wound-dressing patch containing embedded Ag nanoparticles (B). Abbreviation: LSH, linseed hydrogel.

\section{Collagen estimation}

Collagen contents of the regenerated tissue were measured using a reported method. ${ }^{40}$ For this purpose, regenerated tissue of the scratched wound was chopped and suspended into $0.5 \mathrm{M}$ acetic acid $(10 \% \mathrm{w} / \mathrm{v})$ after being washed with $0.5 \mathrm{M}$ sodium acetate. It was stirred for $48 \mathrm{~h}$ and then centrifuged for $2 \mathrm{~h}$ at $5,000 \times \mathrm{g}$. Collagen was precipitated by adding sodium chloride ( $10 \% \mathrm{w} / \mathrm{v})$ and filtered on a weighed Whatman filter paper. Amount of collagen was calculated from the difference in the weight of filter paper before and after filtration.

\section{Statistical analysis}

The data of collagen estimation were expressed as the mean \pm standard deviation (SD) and analyzed using oneway analysis of variance. A $P$-value $<0.05$ was considered statistically significant and denoted by asterisk.

\section{Results and discussion Green synthesis of Ag NPs}

On subjecting the $\mathrm{AgNO}_{3}$ solutions to diffused sunlight, hydrated electrons are generated in the system. These electrons can be used to reduce monovalent silver cations $\left(\mathrm{Ag}^{+}\right)$to zerovalent silver atom $\left(\mathrm{Ag}^{0}\right)$. The $\mathrm{Ag}^{0}$ atoms so generated usually have a size ranging in nanometers. Therefore, UV irradiation can be exploited as a green method for the synthesis of $\mathrm{Ag}$ NPs. This method can also be used to avoid environmentally toxic and hazardous reducing agents. In the present work, we have carried out the green synthesis of Ag NPs using LSH as a green reducing agent under diffused sunlight. The LSH was also evaluated as a storage medium for the synthesized Ag NPs. LSH-mediated synthesis of Ag NPs was carried out using different concentrations of $\mathrm{AgNO}_{3}$ solutions (10, 20, and $30 \mathrm{mmol}$ ). On mixing the reactants, $\mathrm{Ag}^{+}$in the $\mathrm{AgNO}_{3}$ solution reacted with $\mathrm{LSH}$ to give $\mathrm{Ag}(\mathrm{LSH})^{+}$complex. When the mixture was subjected to diffused sunlight, $\mathrm{Ag}^{+}$ in the complex was reduced by LSH to produce $\mathrm{Ag}$ (LSH) precursor. The positive charge on the surface of Ag NPs was electrostatically stabilized and capped by the negative charge on polysaccharide hydroxyls, which resulted in colloidal stabilization of (Ag [LSH]).

The progress of formation of Ag NPs by irradiation of $(\mathrm{Ag}[\mathrm{LSH}])^{+}$complex was followed by noting the color change of reaction mixture with passage of time. The color of LSH and $\mathrm{AgNO}_{3}$ mixture changed from colorless to reddish brown and finally dark brown within $10 \mathrm{~h}$ indicating the complete reduction of $\mathrm{Ag}^{+}$. A schematic illustration of synthesis of LSH-mediated synthesis of Ag NPs is shown in Figure 2. 


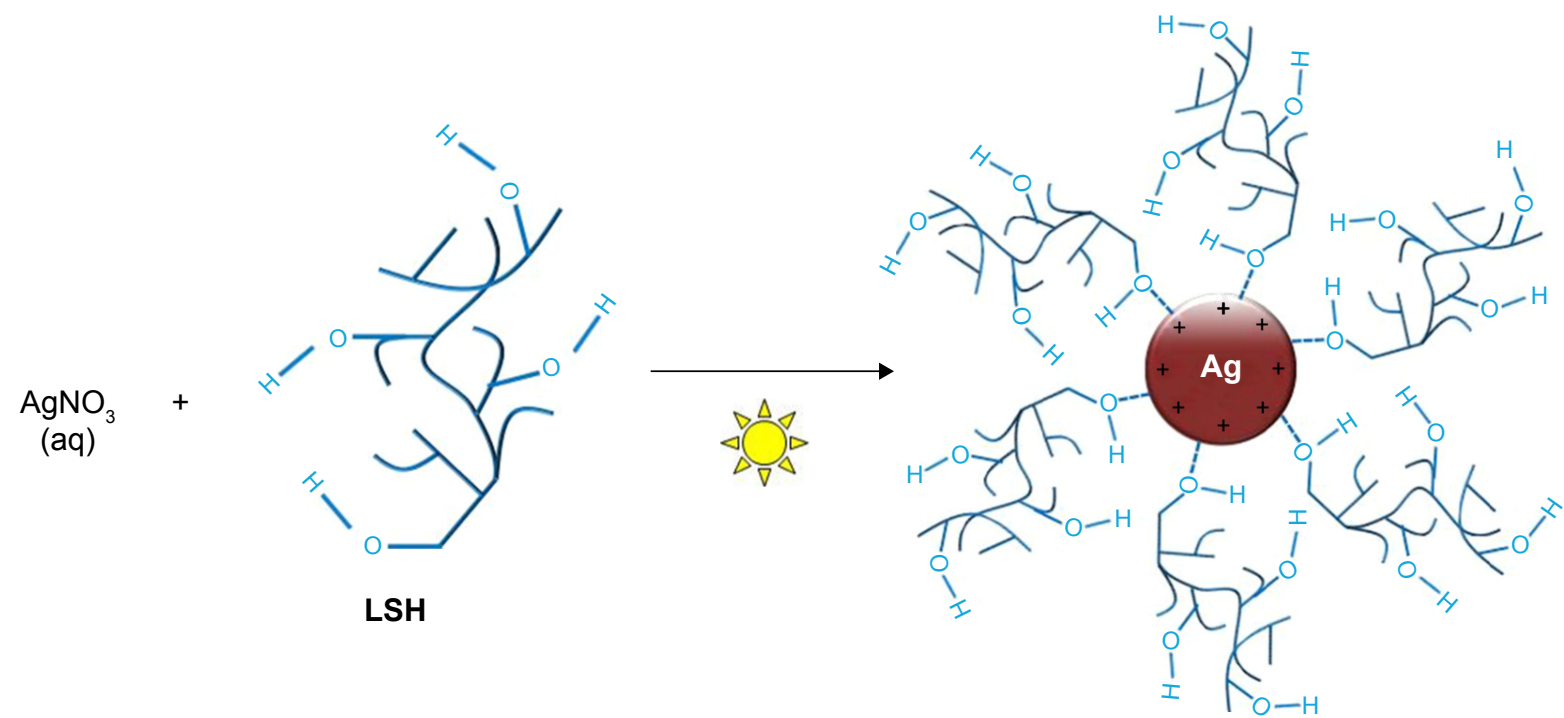

Ag NPs capped by LSH

Figure 2 Schematic illustration showing synthesis of LSH-mediated Ag NPs. Abbreviations: LSH, linseed hydrogel; NPs, nanoparticles.

\section{Characterization of Ag NPs UV/Vis spectrophotometry}

LSH suspension $(2 \mathrm{~mL}$ ) was mixed with different concentrations of $\mathrm{AgNO}_{3}(10,20$, and $30 \mathrm{mmol}, 2 \mathrm{~mL}$ aliquot each) in dark and then placed in diffused sunlight. Reduction of $\mathrm{Ag}^{+}$to $\mathrm{Ag}^{0}$ was indicated by change in color of the reaction mixture and monitored by UV/Vis spectrophotometry. The conduction electrons of Ag NPs exhibit surface plasmon resonance (SPR) because of their collective oscillation, and this SPR results in strong absorption in visible region of spectrum. ${ }^{41}$ The wavelengths absorbed for SPR transitions of Ag NPs are changed by change in reaction time and size of Ag NPs, so reaction mixture showed a color change as the reaction progressed. The color changed from colorless to reddish brown and finally dark brown with passage of time from 0.25 to $10 \mathrm{~h}$. Photographs showing the color change of reaction mixture with progress of reaction are shown in Figure 3.

The LSH-Ag+ solutions showed UV/Vis absorptions at 410, $415,419,425,426,428,430,431$, and $436 \mathrm{~nm}$ for $10 \mathrm{mmol}$, whereas 412, 416, 422, 426, 428, 430, 431, 434, and $436 \mathrm{~nm}$ absorptions were recorded for $20 \mathrm{mmol} \mathrm{LSH}-\mathrm{Ag}^{+}$solution. Likewise, $30 \mathrm{mmol}$ solution showed UV absorptions at 413, $417,424,427,429,432$, 433, 435, and $437 \mathrm{~nm}$. These absorptions were recorded at reaction times of $0.25,0.5,0.75,1$, $2,4,6,8$, and $10 \mathrm{~h}$, respectively. Similar results have been reported in the literature where Ag NPs were synthesized using exogenous reducing agents. ${ }^{42}$ The absorption spectra of Ag NPs showed red shift indicating increase in size of
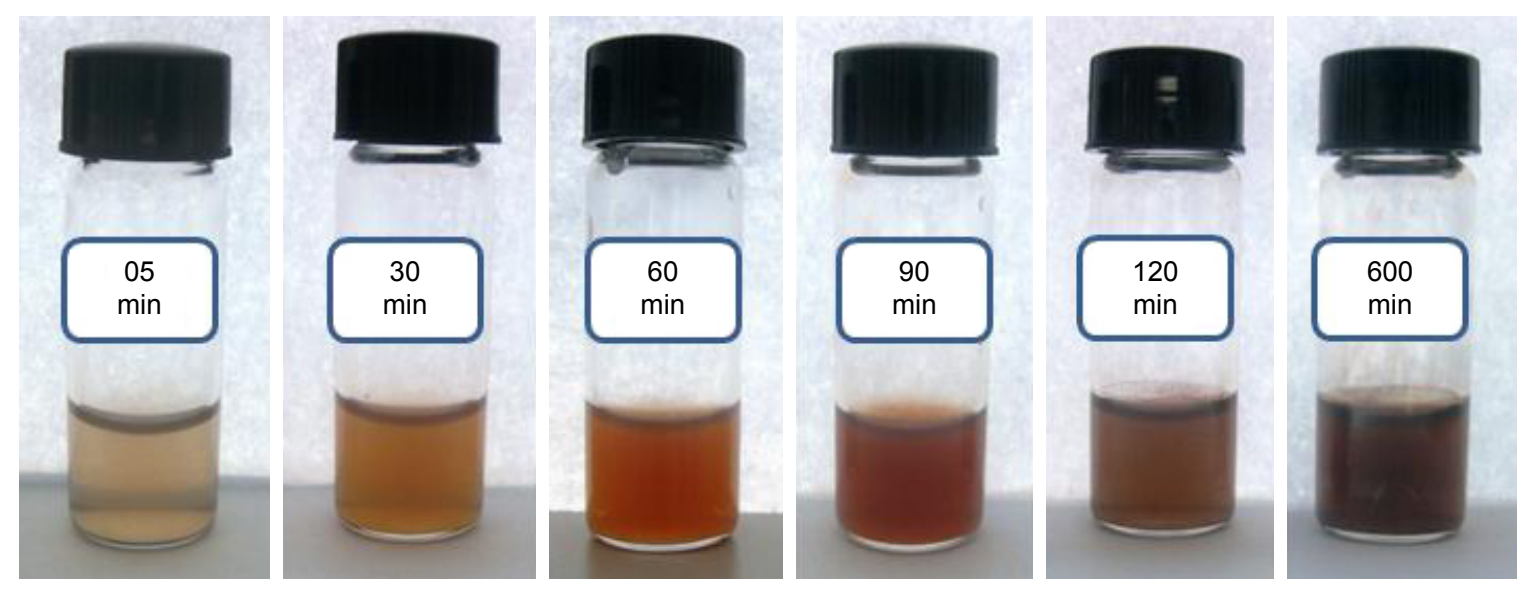

Figure 3 Photographs of linseed hydrogel-Ag+ mixture $\left(20 \mathrm{mmol} \mathrm{AgNO}_{3}\right)$ showing color change with passage of time. Abbreviation: min, minute. 
Ag NPs with increase in reaction time. Absorption intensity also showed an increase when reaction time was increased from 0.25 to $10 \mathrm{~h}$, which indicated continuous reduction of $\mathrm{Ag}^{+}$by LSH with passage of time. Similarly, an increase in absorption intensity was also observed as the concentration of $\mathrm{AgNO}_{3}$ solution was increased from 10 to $30 \mathrm{mmol}$ as evident in Figure 4A-C. This increase in absorption intensity is due to increase in size of the synthesized Ag NPs as shown in TEM images in Figure 5. Results of UV/Vis analyses are summarized in Figure 4.

\section{Effect of temperature on formation of $\mathrm{Ag} \mathrm{NPs}$}

Effect of temperature on rate of formation and size of LSHmediated Ag NPs was also noted. $\operatorname{AgNO}_{3}(10,20$, and $30 \mathrm{mmol}$ ) solution was mixed with LSH solution and
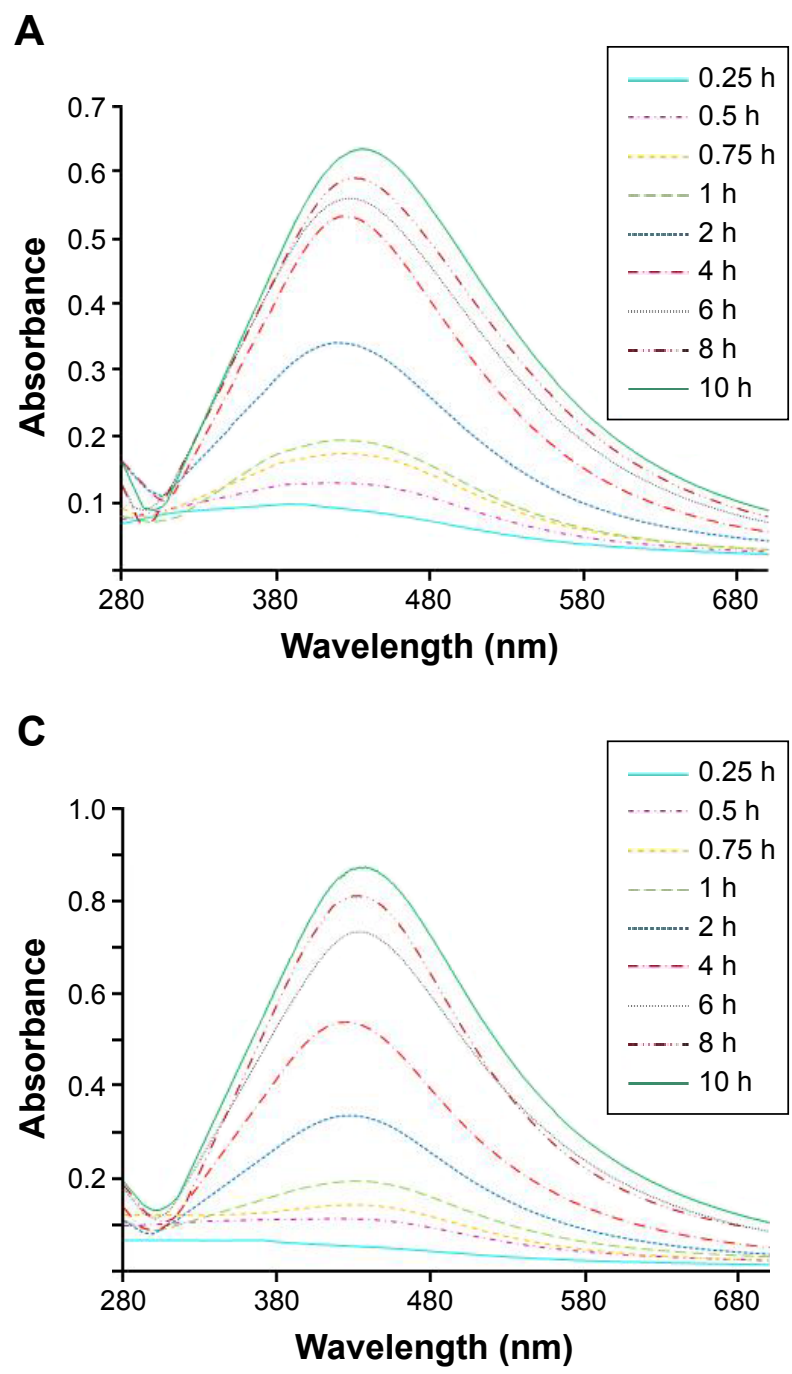

progress of reaction was monitored at different temperatures $\left(50,75\right.$, and $\left.100^{\circ} \mathrm{C}\right)$. This experiment was performed in dark to avoid any photochemical reaction. Comparison of absorption bands showed that Ag NPs exhibit higher absorption with increase in temperature (Figure 6). The increase in absorption wavelength for a given reaction time at higher temperature indicated that rate of formation of Ag NPs and size of synthesized NPs are dependent on the temperature. These findings are in line with the observations by other scientists. ${ }^{43}$

\section{TEM of isolated Ag NPs}

Size distribution and morphology of Ag NPs were assessed by TEM. Ag NPs were separated from LSH and $\mathrm{AgNO}_{3}$ (10, 20, and $30 \mathrm{mmol})$ solutions by centrifugation, and TEM image was recorded. TEM images confirmed the formation

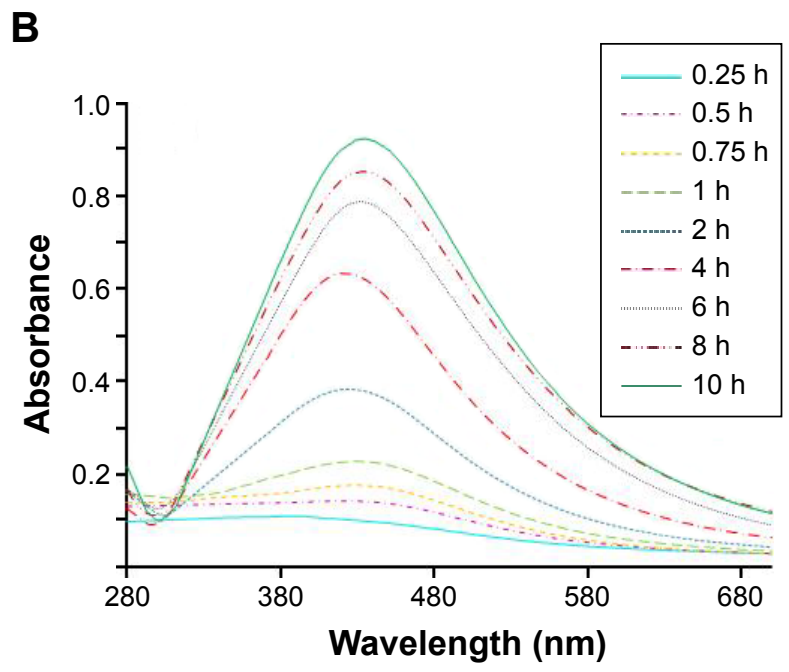

D

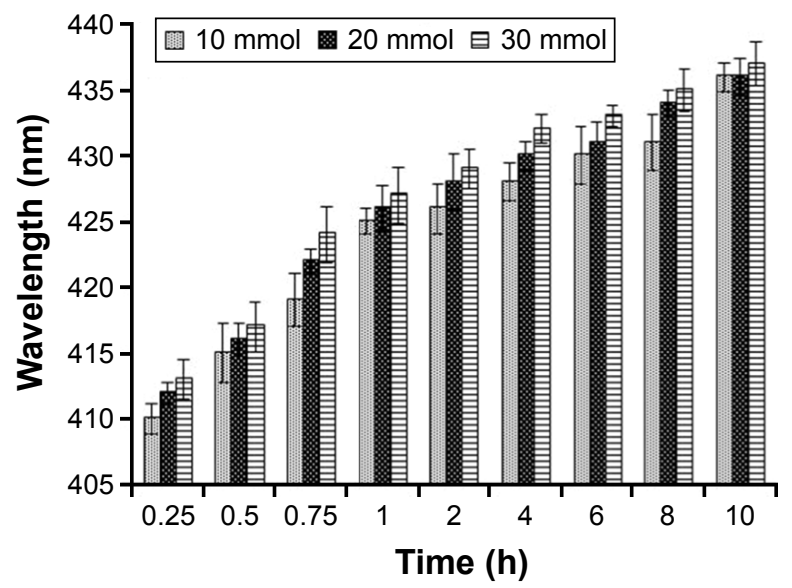

Figure 4 Ultraviolet-visible spectra of linseed hydrogel-mediated $\mathrm{Ag}$ NPs: $10 \mathrm{mmol}(\mathbf{A}), 20 \mathrm{mmol}(\mathbf{B})$, and $30 \mathrm{mmol}$ solution of $\mathrm{AgNO}_{3}(\mathbf{C})$ at different reaction times and cumulative graphical representation (D) showing increase in absorption of $\mathrm{Ag} N \mathrm{~N}$ solutions with increase in concentration and reaction time. Abbreviations: h, hour; NPs, nanoparticles. 

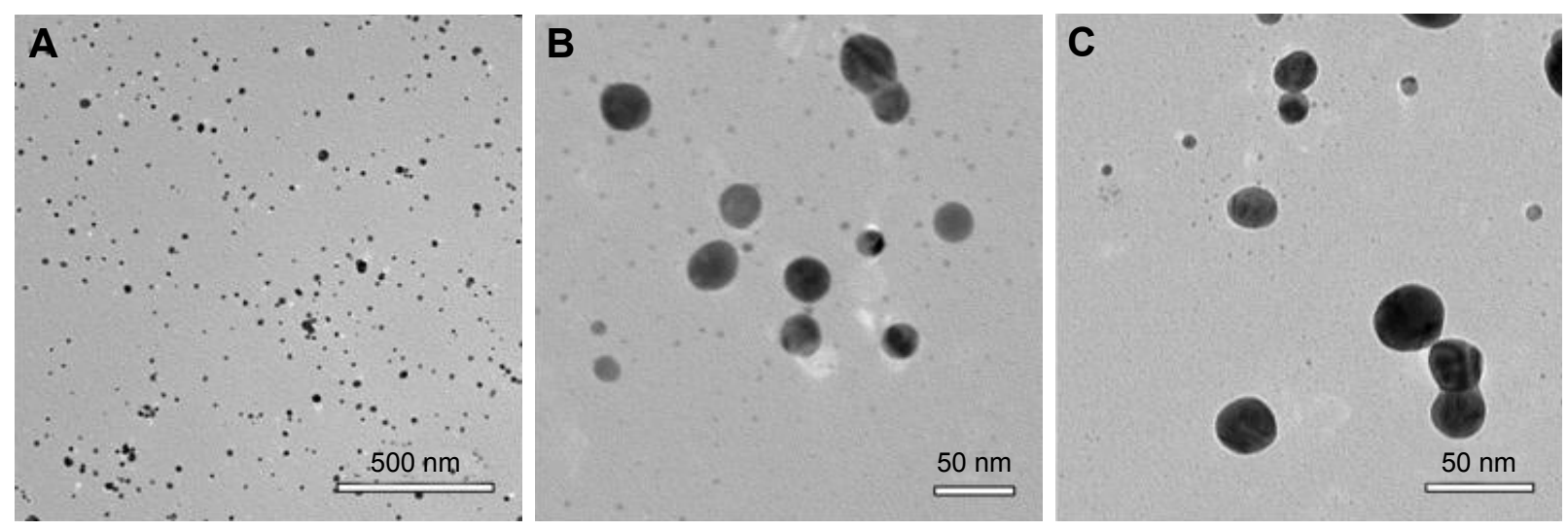

Figure 5 Transmission electron microscopy images of Ag nanoparticles isolated from (A) 10 (I0-25 nm), (B) 20 (I0-30 nm), and (C) $30 \mathrm{mmol}$ (I0-35 nm) linseed hydrogel-

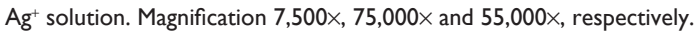

of spherical Ag NPs in the size regimen of 10-25, 10-30, and $10-35 \mathrm{~nm}$ for 10,20 , and $30 \mathrm{mmol} \mathrm{AgNO}_{3}$ solution (Figure 5), respectively. For film formation, a mixture of $\mathrm{LSH}$ and $\mathrm{AgNO}_{3}(20 \mathrm{mmol})$ solution was air dried in dark and stored for 6 months. After selected storage period, the film was dissolved in deionized water and Ag NPs were isolated by centrifugation. TEM image of isolated Ag NPs showed that the NPs retained their morphology and size over the storage period. Therefore, it was inferred that the LSH thin film could be used as storage media for Ag NPs.

\section{Powder X-ray diffraction}

PXRD (Figure 7) was used to confirm the crystal phase of the isolated $\mathrm{Ag}$ NPs in the range of $10^{\circ} \mathrm{C}-80^{\circ} \mathrm{C}, 2 \theta$. The diffraction peaks centered at (111), (200), (220), and (311) indicated that Ag NPs had face-centered cubic lattice in all samples. Diffraction pattern was also recorded for the Ag NPs isolated from stored thin films (6 months). It was revealed that there was no change in diffraction peak of samples after

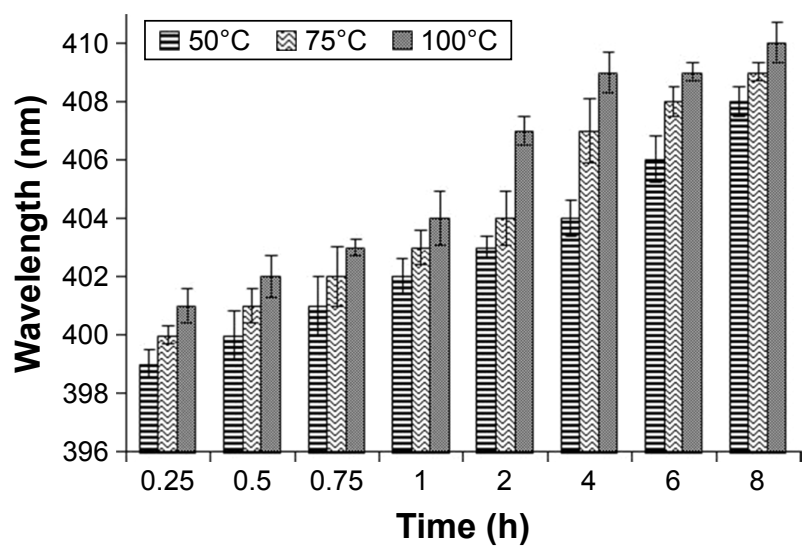

Figure 6 Ultraviolet-visible spectroscopy absorptions of Ag nanoparticles obtained from $\mathrm{AgNO}_{3}$ solution $(10 \mathrm{mmol})$ at different temperatures and reaction times. Abbreviation: h, hour. storage. Therefore, it was concluded that LSH could be used for the long-term storage of Ag NPs without affecting their morphology.

\section{Storage of Ag NPs in LSH thin films}

Potential of LSH for the storage of Ag NPs in solution and in the form of a thin film was evaluated. For this purpose, $\mathrm{Ag}$ NPs were synthesized by reducing the $\mathrm{AgNO}_{3}(20 \mathrm{mmol})$ solution with LSH over $10 \mathrm{~h}$. UV/Vis spectra were recorded

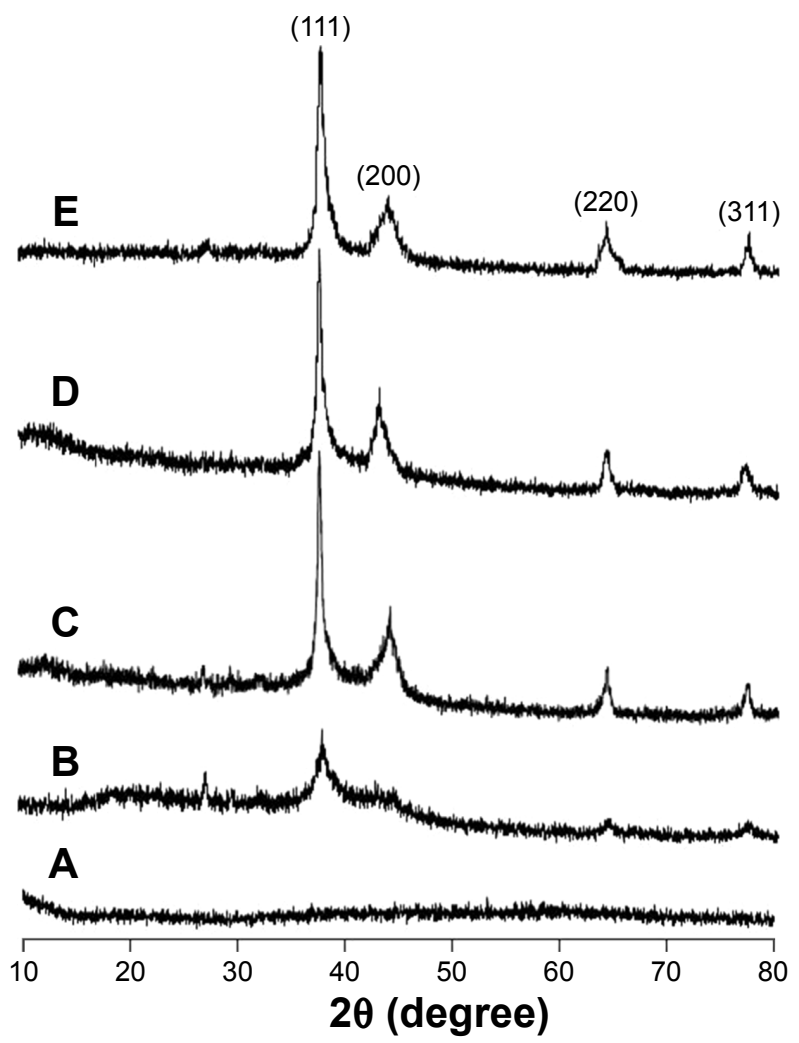

Figure 7 Powder X-ray diffraction spectra: LSH (A), Ag NPs-embedded LSH film (B), and isolated Ag NPs, $10 \mathrm{mmol}$ (C), $20 \mathrm{mmol}$ (D), and $30 \mathrm{mmol}$ (E). Abbreviations: LSH, linseed hydrogel; NPs, nanoparticles. 
after 10 and $24 \mathrm{~h}$, and the sample was stored as dry thin film in dark. Absorption spectra of the isolated Ag NPs were acquired by dissolving the films in deionized water. Comparable absorption spectra were obtained after the storage period (15 days, 30 days and 6 months). No significant change in the absorption wavelength and intensity was observed for the stored samples. Therefore, it was inferred that the Ag NPs did not undergo agglomeration on drying and storage in the form of thin films. TEM images of isolated Ag NPs from stored thin film (6 months) were recorded and observed uniformed size and shape as of freshly prepared Ag NPs. Results of storage experiments are illustrated in Figure 8.

\section{Antimicrobial activity of Ag NPs}

LSH-based Ag NPs were tested for antimicrobial properties. Significant antibacterial and antifungal activity was observed against $S$. mutans, $S$. epidermidis, $P$. aeruginosa, E. coli, S. aureus, B. subtilis, A. odontolyticus, and A. niger strains and showed inhibition zones of 22, 13, 18, 09, 20, 15, 16 , and $10 \mathrm{~mm}$, respectively, on being exposed to the aqueous solution of $\mathrm{Ag}$ NPs synthesized with $\mathrm{AgNO}_{3}(20 \mathrm{mmol})$ solution. No antimicrobial activity was noticed for control experiments performed by using LSH solution and distilled water; however, $\mathrm{AgNO}_{3}(20 \mathrm{mmol})$ solution was found active against the above-mentioned strains as shown in Figure 9. Antimicrobial properties of Ag NPs prepared from 10 to $30 \mathrm{mmol} \mathrm{AgNO}_{3}$ solution were also tested. Results of antimicrobial activity of Ag NPs $(20 \mathrm{mmol})$ are summarized in a bar graph in Figure 9, and the average of three readings is reported. As a typical example, antimicrobial activity of Ag NPs (20 mmol) against $P$. aeruginosa is shown in Figure 9. It is revealed that LSH-based Ag NPs had a clear zone of inhibition against the above-mentioned strains, while LSH alone did not show any effect on the microbial cultures.

\section{Wound-healing studies}

To evaluate wound-healing properties of LSH films impregnated with Ag NPs, excision wounds were created on rear leg of the rabbit. Average wound diameter was calculated for different groups of rabbits. Wound healing was estimated

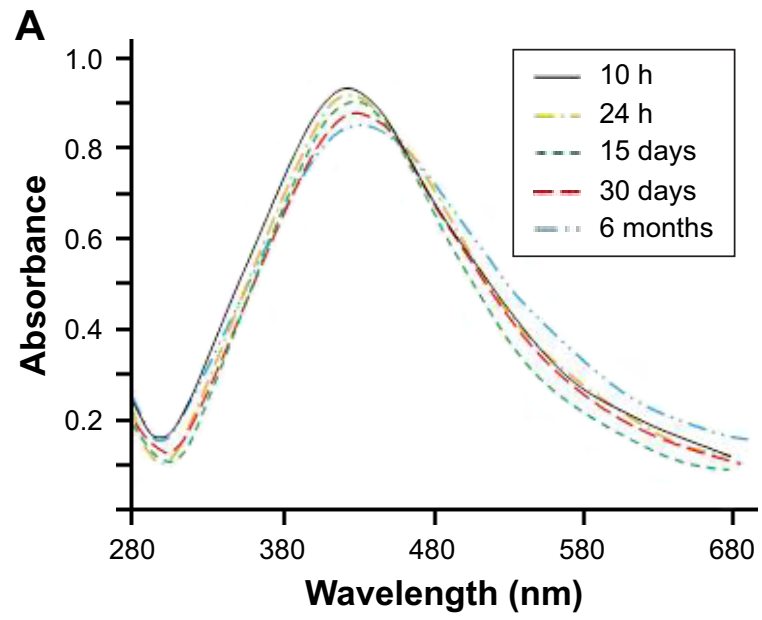

B
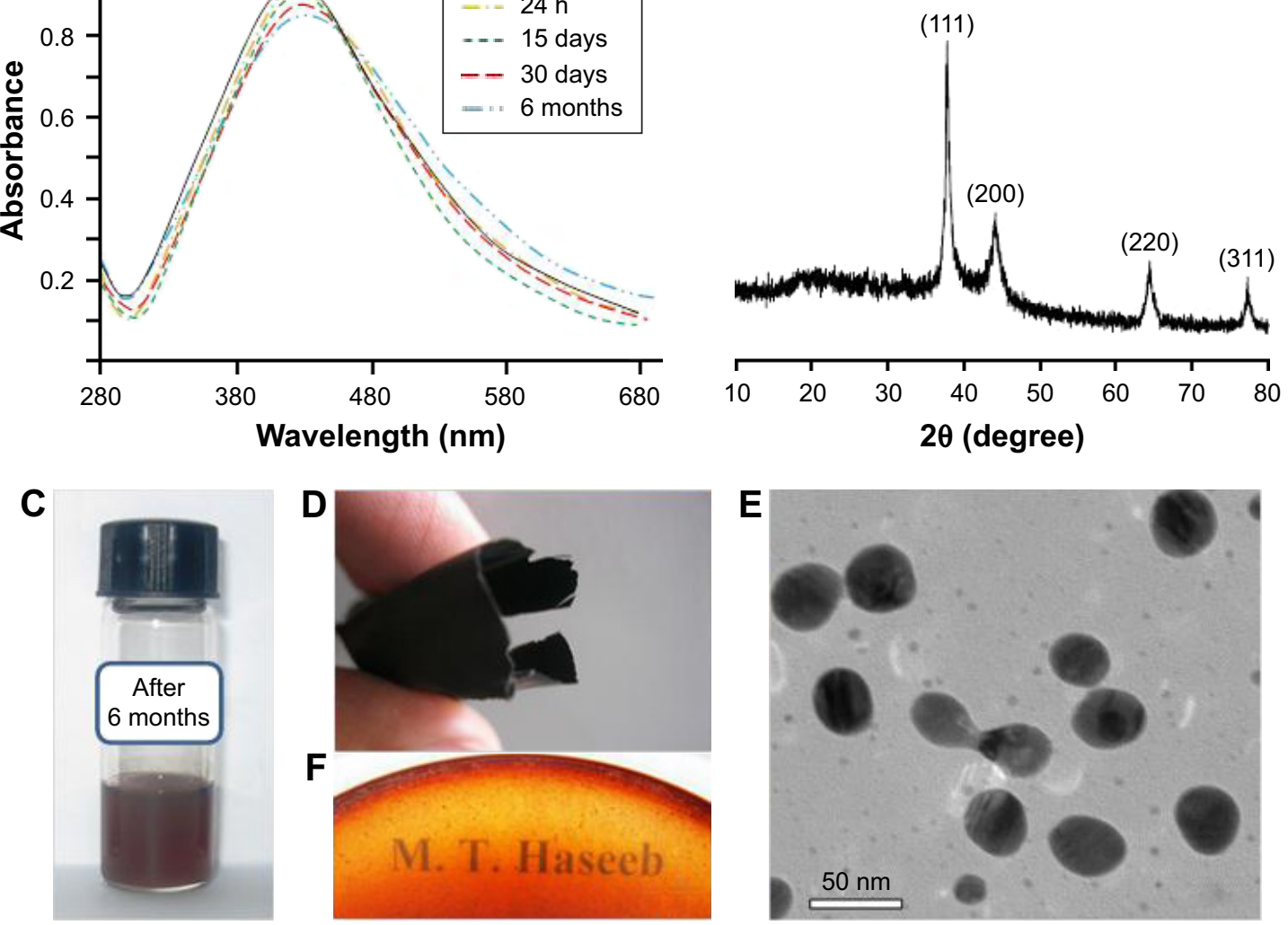

Figure 8 Ultraviolet-visible spectra of $\mathrm{AgNO}_{3}(20 \mathrm{mmol})$ solution in LSH recorded after $10 \mathrm{~h}, 24 \mathrm{~h}, 15$ days, 30 days, and 6 months storage (A), powder X-ray diffraction spectrum of $\mathrm{Ag} \mathrm{NPs}$ isolated after 6 months storage of $\mathrm{LSH}-\mathrm{Ag}$ NP film (B), vial containing solution of stored film in water (C), foldable and see-through Ag NPs-embedded LSH thin film (D and F), respectively, and transmission electron microscopy image of isolated Ag NPs (10-30 nm) from thin films after 6-month storage in dark (E). Magnification 75,000x.

Abbreviations: LSH, linseed hydrogel; NPs, nanoparticles. 
A

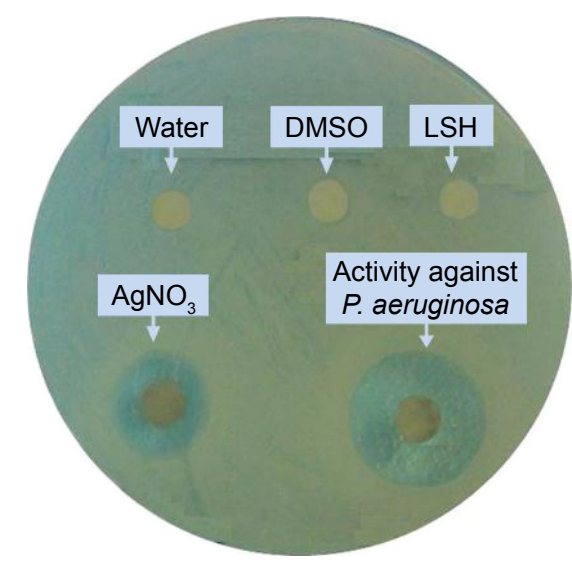

B

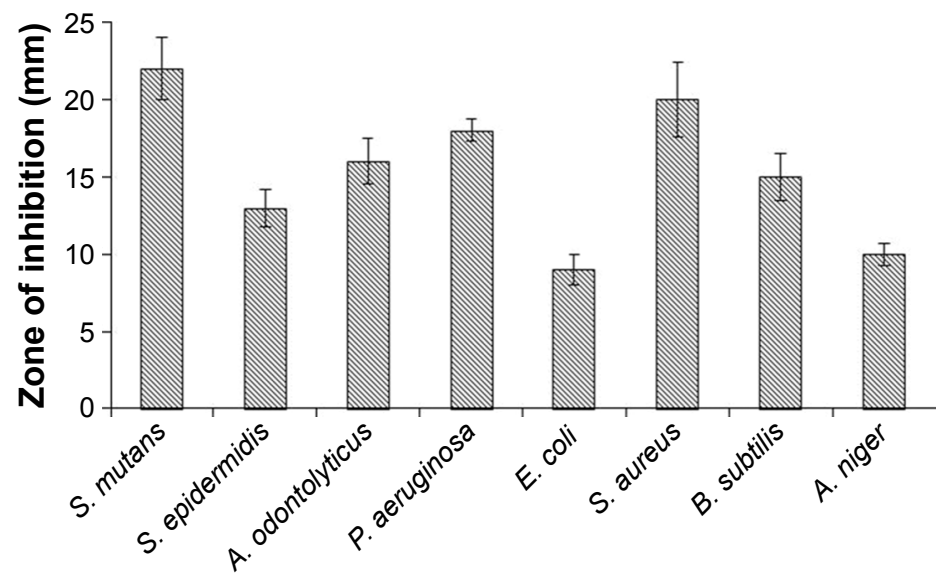

Microorganisms

Figure 9 Photograph indicating the antimicrobial activity of $\mathrm{Ag}$ nanoparticles (20 mmol) against $P$. aeruginosa along with negative and positive control (A) and measurements of zone of inhibition of different bacterial and fungal strains (B).

Abbreviations: A. niger, Aspergillus niger; A. odontolyticus, Actinomyces odontolyticus; B. subtilis, Bacillus subtilis; DMSO, dimethyl sulfoxide; E. coli, Escherichia coli; LSH, linseed hydrogel; P. aeruginosa, Pseudomonas aeruginosa; S. aureus, Staphylococcus aureus; S. epidermidis, Staphylococcus epidermidis; S. mutans, Streptococcus mutans.

from percentage closure of wound area $\left(\mathrm{mm}^{2}\right)$ noted at different times after wound induction. Control group showed a wound closure of $0.25,1.11,20.32,38.76,62.55$, and $83.27 \%$ on 1 st, $3 \mathrm{rd}$, 6th, 9th, 12th, and 15 th day, respectively. The animals treated with standard Band aid dressing showed closure area of 2.17, 19.45, 63.67, 87.27, 98.49, and $100 \%$, while the test group showed $1.41,16.73,54.62$, 93.21, 99.22, and 100\% wound closure on 1st, 3rd, 6th, 9th, 12 th, and 15 th day, respectively. Results indicated that tissue regeneration and percentage wound closure of LSH-based wound dressing were comparable to the standard Band aid dressing. Results of wound-healing study for various groups are summarized in Table 1.

To understand the mechanism of wound healing, collagen content and tensile strength of the epithelialized wound tissue were measured. The control group showed a collagen content of $38 \mathrm{mg} / \mathrm{kg}$ while standard and test groups showed a collagen content of 69 and $65 \mathrm{mg} / \mathrm{kg}$, respectively. Therefore, it was noticed that the standard and test groups had higher collagen content than the control group, which resulted in rapid wound healing in the said groups in contrast to the control. Tensile strength was also found higher for test and standard groups as compared to the control. Results of collagen content of regenerated tissue for various groups are depicted in Figure 10. It was inferred that collagen synthesis is initiated at the wound area through formation of a polypeptide precursor. Inter- and intramolecular cross-linking of this collagen then results in rapid tissue regeneration and wound closure.

\section{Conclusions}

Herein, we report on a cost-effective and eco-friendly method for the synthesis of Ag NPs using biodegradable and nontoxic $\mathrm{LSH}$ as a template nanoreactor and reducing agent. Effect of $\mathrm{AgNO}_{3}$ concentration, reaction time, and change in temperature on the size and distribution of $\mathrm{Ag}$ NPs has been studied. Narrow size distribution and uniform dispersion of Ag NPs were achieved in the presence of LSH as a green reducing agent. LSH thin films proved to be an excellent storage medium to prevent agglomeration of $\mathrm{Ag}$ NPs with passage of time. Ag NPs-embedded LSH films can be exploited for potential biomedical and antimicrobial applications. Exploration of catalytic properties and surfaceenhanced Raman scattering of Ag NPs are future prospects of the study.

Table I Wound diameter and wound closure \% after selected day intervals

\begin{tabular}{|c|c|c|c|c|c|c|}
\hline & \multicolumn{6}{|c|}{ Wound diameter in $\mathrm{mm} \pm$ standard deviation (\% of wound closure) at day } \\
\hline & Ist & 3rd & 6th & 9th & 12th & 15th \\
\hline Control & $5.98 \pm 0.06(0.25)$ & $5.93 \pm 0.05(1.11)$ & $4.78 \pm 0.03(20.32)$ & $3.67 \pm 0.10(38.76)$ & $2.25 \pm 0.06(62.55)$ & $1.00 \pm 0.02(83.27)$ \\
\hline Band aid ${ }^{\circledR}$ dressing & $5.87 \pm 0.04(2.17)$ & $4.83 \pm 0.08(19.45)$ & $2.18 \pm 0.09(63.67)$ & $0.76 \pm 0.05(87.27)$ & $0.09 \pm 0.08(98.49)$ & $(100)$ \\
\hline Linseed hydrogel dressing & $5.9 I \pm 0.04(I .4 I)$ & $4.94 \pm 0.07(17.73)$ & $2.72 \pm 0.08(54.62)$ & $0.4 I \pm 0.06(93.2 I)$ & $0.05 \pm 0.08(99.22)$ & $(100)$ \\
\hline
\end{tabular}




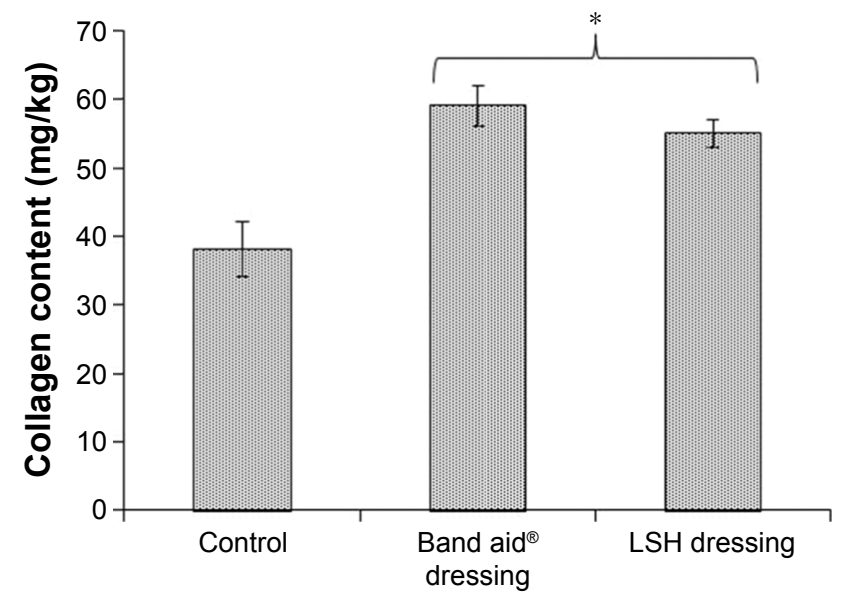

Figure 10 Collagen contents of epithelialized wound tissue of various groups after I5th day.

Note: Statistical significance from control group and is shown by $* P<0.05$.

Abbreviation: LSH, linseed hydrogel.

\section{Acknowledgment}

MT Haseeb gratefully acknowledges Higher Education Commission (HEC) of Pakistan for providing the financial support under "HEC Indigenous 5000 Fellowship" and International Research Support Initiative Program.

\section{Disclosure}

The authors report no conflicts of interest in this work.

\section{References}

1. Elghanian R, Storhoff JJ, Mucic RC, Letsinger RL, Mirkin CA. Selective colorimetric detection of polynucleotides based on the distancedependent optical properties of gold nanoparticles. Science. 1997; 277(5329):1078-1081.

2. Kamat PV. Photophysical, photochemical and photocatalytical aspects of metal nanoparticles. J Phys Chem B. 2002;106:7729-7744.

3. Mallik K, Witcomb MJ, Scurell MS. Redox catalytic property of gold nanoclusters: evidence of an electron-relay effect. Appl Phys A. 2005; 80:797-801

4. Liz-Marzan L. Tailoring surface plasmons through the morphology and assembly of metal nanoparticles. Langmuir. 2006;22(1):32-41.

5. Wang S, Riahi R, Li N, Zhang DD, Wong PK. Single cell nanobiosensors for dynamic gene expression profiling in native tissue microenvironments. Adv Mater. 2015;27(39):6034-6038.

6. Wang S, Sun J, Zhang DD, Wong PK. A nanobiosensor for dynamic single cell analysis during microvascular self-organization. Nanoscale. 2016;8(38):16894-16901.

7. Cao YC, Jin R, Nam J, Thaxton CS, Mirkin CA. Raman-dye-labeled nanoparticle probes for proteins. J Am Chem Soc. 2003;125(48): 14676-14677.

8. Xia A, Rogers JA, Paul KE, Whitesides GM. Unconventional methods for fabricating and pattering nanostructures. Chem Rev. 1999;99(7): 1823-1848.

9. Deheer WA. The physics of simple metal clusters: experimental aspects and simple models. Rev Mod Phys. 1993;65:611-676.

10. Littau KA, Szajowski PJ, Muller AJ, Kortan AR, Brus LE. A luminescent silicon nanocrystal colloid via a high-temperature aerosol reaction. J Phys Chem. 1993;97:1224-1230.

11. Lee AF, Baddeley CJ, Hardacre C, et al. Structural and catalytic properties of novel Au/Pd bimetallic colloid particles: EXAFS, XRD and acetylene coupling. J Phys Chem. 1995;99:6096-6102.
12. Schimd G. Large clusters and colloids metals in the embryonic state. Chem Rev. 1992;92:1709-1727.

13. Krutyakov YA, Kudrynskiy AA, Olenin AY, Lisichkin GV. Synthesis and properties of silver nanoparticles: advances and prospects. Russ Chem Rev. 2008;77:233-257.

14. Sarkar J, Saha S, Chattopadhyay D, Patra S, Acharya K. Mycosynthesis of silver nanoparticles and investigation of their antimicrobial activity. J Nanosci Nanoeng App. 2011;1:17-26.

15. Bosetti M, Masse A, Tobin E, Cannas M. Silver coated materials for external fixation devices: in vitro biocompatibility and genotoxicity. Biomaterials. 2002;23(3):887-892.

16. Hu Z, Zhang J, Chan WL, Szeto YS. Suspension of silver oxide nanoparticles in chitosan solution and its antibacterial activity in cotton fabrics. MRS Proceedings. 2006;920:S02-S03.

17. Rivas L, Sanchez-cartos S, Garcia-Ramos JV, Marcillo G. Growth of silver colloidal particles obtained by citrate reduction to increase the Raman enhancement factor. Langmuir. 2001;17:574-577.

18. Malune A, Kohon JY, Takeda Y, Kondow T. Structure and stability of silver nanoparticles in aqueous solution produced by laser ablation. J Phys Chem B. 2000;104:8333-8337.

19. Zhu JJ, Liu SW, Palchik O, Kottypin Y, Gedanken A. Shape-controlled synthesis of silver nanoparticles by pulse sonoelectrochemical methods. Langmuir. 2000;16:6396-6399.

20. Wang S, Sun J, Xiao Y, Lu Y, Zhang DD, Wong PK. Intercellular tension negatively regulates angiogenic sprouting of endothelial tip cells via Notch1-Dl14 signaling. Adv Biosystems. 2017;1(1-2).

21. Riahi R, Wang S, Long M, et al. Mapping photothermally induced gene expression in living cells and tissues by nanorod-locked nucleic acid complexes. ACS Nano. 2014;8(4):3597-3605.

22. Chen M, Wang LY, Han JT, Zhang JY, Li ZY, Qian DJ. Preparation and study of polyacrylamide-stabilized silver nanoparticles through a one-pot process. J Phys Chem B. 2006;110(23):11224-11231.

23. Kuo PL, Chen WF. Formation of silver nanoparticles under structured amino groups in pseudo-dendritic poly (allylamine) derivatives. $J$ Phys Chem B. 2003;107:11267-11272.

24. Pastoriza-Santos I, Liz-Marzan LM. Formation of PVP-protected metal nanoparticles in DMF. Langmuir. 2002;18:2888-2894.

25. Zou X, Bao H, Guo H, et al. Mercaptoethane sulfonate protected, water-soluble gold and silver nanoparticles: syntheses, characterization and their building multilayer films with polyaniline via ion-dipole interactions. J Colloid Interface Sci. 2006;295(2):401-408.

26. Popa M, Pradell T, Crespo D, Calderón-Moreno JM. Stable silver colloidal dispersions using short chain polyethylene glycol. Colloids Surfaces A Physicochem Eng Asp. 2007;303:184-190.

27. Vimala K, Sivudu KS, Mohan YM, Sreedhar B, Raju KM. Controlled silver nanoparticles synthesis in semi-hydrogel networks of poly (acrylamide) and carbohydrates: a rational methodology for antibacterial application. Carbohydr Polym. 2009;75:463-471.

28. Murphy CJ. Sustainability as an emerging design criterion in nanoparticle synthesis and applications. J Mater Chem. 2008;18:2173-2176.

29. Prozorova GF, Pozdnyakov AS, Kuznetsova NP, et al. Green synthesis of water-soluble nontoxic polymeric nanocomposites containing silver nanoparticles. Int J Nanomed. 2014;9:1883-1889.

30. Muzzarelli RAA. Genipin-crosslinked chitosan hydrogels as biomedical and pharmaceutical aids. Carbohydr Polym. 2009;77:1-9.

31. Hebeish AA, El-Rafie MH, Abdel-Mohdy FA, Abdel-Halim ES, Emam HE. Carboxymethylcellulose for green synthesis and stabilization of silver nanoparticles. Carbohydr Polym. 2010;82(3):933-941.

32. Angelova T, Rangelova N, Yuryev R, Georgieva N, Müller R. Antibacterial activity of $\mathrm{SiO}_{2}$ /hydroxypropylcellulose hybrid materials containing silver nanoparticles. Mater Sci Eng A. 2012;32(5):1241-1246.

33. Hussain MA, Shah A, Jantan I, et al. One pot light assisted green synthesis, storage and antimicrobial activity of dextran stabilized silver nanoparticles. J Nanobiotechnol. 2014;12:53-58.

34. Huang H, Yang X. Synthesis of polysaccharide-stabilized gold and silver nanoparticles: a green method. Carbohydr Res. 2004;339(15): $2627-2631$. 
35. Long DW, Wu GZ, Chen SM. Preparation of oligochitosan stabilized silver nanoparticles by gamma irradiation. Rad Phys Chem. 2007;76(7): $1126-1131$.

36. Cui W, Mazza G, Biliaderis CG. Chemical structure, molecular size distributions and rheological properties of flaxseed gum. J Agric Food Chem. 1994;42:1891-1895.

37. Muralikrishna G, Salimath PV, Tharanathan RN. Structural features of an arabinoxylan and a rhamno-galacturonan derived from linseed mucilage. Carbohydr Res. 1987;161(2):265-271.

38. Haseeb MT, Hussain MA, Yuk SH, Bashir S, Nauman M. Polysaccharides based superabsorbent hydrogel from Linseed: dynamic swelling, stimuli responsive on-off switching and drug release. Carbohydr Polym. 2016;136:750-756.

39. Bauer AW, Kirby WM, Sherris JC, Turck M. Antibiotic susceptibility testing by a standardized single disk method. Am J Clin Pathol. 1966; 45(4):493-496.
40. Lee KH, Tong TG. Study on the mechanism of action of salicylates, retardation of wound healing by aspirin. J Pharm Sci. 1968;57(6): 1042-1046.

41. Mock JJ, Barbic M, Smith DR, Schultz DA, Schultz S. Shape effects in plasmon resonance of individual colloidal silver nanoparticles. J Chem Phys. 2002;116:6755-6759.

42. Hussain MA, Shah A, Jantan I, et al. Hydroxypropylcellulose as a novel green reservoir for the synthesis, stabilization, and storage of silver nanoparticles. Int J Nanomed. 2015;10:2079-2088.

43. Amin M, Iram F, Iqbal MS, Saeed MZ, Raza M, Alam S. Arabinoxylanmediated synthesis of gold and silver nanoparticles having exceptional high stability. Carbohydr Polym. 2013;92(2):1896-1900.
International Journal of Nanomedicine

\section{Publish your work in this journal}

The International Journal of Nanomedicine is an international, peerreviewed journal focusing on the application of nanotechnology in diagnostics, therapeutics, and drug delivery systems throughout the biomedical field. This journal is indexed on PubMed Central, MedLine, CAS, SciSearch $®$, Current Contents $® /$ Clinical Medicine,

\section{Dovepress}

Journal Citation Reports/Science Edition, EMBase, Scopus and the Elsevier Bibliographic databases. The manuscript management system is completely online and includes a very quick and fair peer-review system, which is all easy to use. Visit http://www.dovepress.com/ testimonials.php to read real quotes from published authors.

Submit your manuscript here: http://www.dovepress.com/international-journal-of-nanomedicine-journal 